\title{
HACIA UN SISTEMA DE PENSIONES PERUANO COMPETITIVO
}

\author{
TOWARDS A COMPETITIVE PERUVIAN PENSION SYSTEM \\ ManuelAib erto Hidaigo Tup ia * \\ Em ino Gustavo Bob bio Rosas* \\ Aib ERTo ESP INOZA VAIENZUEla* \\ Docentes Auxiliares de la Facultad de Ciencias Contables \\ Universidad Nacional Mayor de San Marcos-UNMSM / Lima-Perú \\ David Juan SÁNCHEZ CRUZ** \\ Docente Contratado de la Facultad de Ciencias Contables \\ Universidad Nacional Mayor de San Marcos-UNMSM / Lima-Perú \\ [Recepción: agosto 2016/ Conformidad: setiembre 2016]
}

\section{RESUMEN}

El estudio recoge las percepciones sobre el sistema de pensiones peruano respecto de la posibilidad de optimizar la gestión financiera de los recursos del Fondo Nacional de Pensiones, tendiente a crear una mayor rentabilidad y la competitividad con respecto al sistema en su conjunto, generando una alternativa real frente al sistema privado de pensiones.

Se realizó el análisis documental y se aplicaron encuestas a tres grupos de informantes: sector social organizado, especialistas académicos, y responsables del sistema de pensiones.

El problema de investigación fue:

¿De qué manera la intervención de la ONP con profesionalización de inversiones, incide en la competitividad del Sistema Pensiones en el Perú?

Los resultados obtenidos de la aplicación de las encuestas a especialistas y los representantes del sector social fueron que la profesionalización de las inversiones de la ONP es posible y deseable, requiriendo ello cambios normativos y administrativos, y que se debería aplicar conforme a la teoría de inversiones en portafolio; y que, dadas las condiciones, generaría una mayor competitividad en el sistema previsional en su conjunto, impactando positivamente en la estabilidad y crecimiento del mercado de capitales y de la economía nacional, e impactando positivamente en el bienestar de los pensionistas y aportantes.

\section{Palabras Clave:}

ONP, Sistema Nacional de Pensiones, Sistema Privado de Pensiones, Mercado de Valores, SBS.

\begin{abstract}
The study gathers perceptions about the Peruvian pension system regarding the possibility of optimizing the financial management of the resources of the National Pension Fund, aiming to create a greater profitability and competitiveness with respect to the system, generating a real alternative the private pension system. A documentary analysis was carried out and surveys were applied to three groups of informants: organized social sector, academic specialists, and pension system managers.

The research problem was: How does the intervention of the ONP (National Pension Bureau) with professionalization of investments affects the competitiveness of the Pension System in Peru?

The results obtained from the application of the surveys to specialists and representatives of the social sector were that the professionalization of ONP investments is possible and desirable, requiring regulatory and administrative changes and that should be applied according to the investment theory In portfolio. Plus, given the conditions, that scenario would generate greater competitiveness in the pension system as a whole, positively impacting the stability and growth of the capital market and the national economy, also positively impacting the welfare of pensioners and contributors.
\end{abstract}

\section{Keywords:}

ONP, National Pension System, Private Pension System, Stock Market, SBS

\footnotetext{
* Magister en Educación - Economista. Email: hidalgoman@gmail.com

* Doctor en Políticas Públicas, con mención en Defensa Nacional y desarrollo Sostenible - UAP. Email: emilio_bobbio19@hotmail.com

* Doctor en Ciencias Contables y Empresariales de la UNMSM. Economista y Contador. Email: aespinozav@unmsm.edu.pe

** Magister en Economía - UNMSM. Email: davidsanch_c@hotmail.com
} 


\section{INTRODUCCIÓN}

El Sistema de Pensiones en el Perú es un tema amplio, complejo y urgente, debido a las siguientes circunstancias:

- La gran cantidad de personas involucradas en forma vital,

- La poca cobertura del sistema

- Las pensiones escasas logradas tanto en el Sistema Nacional como en el Sistema Privado;

- Los retos a la sostenibilidad del sistema, debido al financiamiento público creciente en el Sistema Nacional, y la insuficiencia de los aportes en el Sistema Privado.

Todo ello ha causado que existan una serie de iniciativas, parciales o globales, para reformar el sistema, de todo signo: desde las reformas más privatistas, tendientes a disminuir el sistema público a efectos de reducir el gasto presupuestal; hasta las reformas restrictivas del sistema privado y una vuelta al financiamiento público y la gestión pública de las pensiones. Tal es el caso de Cruz - Saco, Mendoza y Seminario (2014), Mendiola y Aguirre (2013); Alonso, Sánchez y Tuesta (2014), Valdivieso (2014), entre otros.

Durante el estudio se discutió en el Congreso Nacional, aprobándose una reforma parcial para permitir el retiro de una parte sustantiva de la pensión del afiliado jubilado al Sistema Privado de Pensiones, lo cual antes era restringido.

Sin embargo, la investigación se centró sólo en un aspecto de toda la problemática, aspecto que, a pesar de todo, presenta un gran potencial de beneficios; claro esta, cumpliendo ciertas condiciones. El criterio era identificar si el uso del Fondo administrado por la ONP (sistema público), podría ser incrementado, a tal fin, que pueda generar los retornos necesarios para:

1.Mejorar la rentabilidad del fondo, incrementando las pensiones pagadas a los pensionistas de la ONP.

2.Reducir, a largo plazo, la carga presupuestal.

3.Crear una factibilidad mayor para la ONP.

4.Incrementar la competitividad general del Sistema.

La propuesta de la "profesionalización de las inversiones de la ONP”, se basa en la experiencia previa del Fondo Complementario de Reservas de la ONP, que en su momento, dio muy buenos resultados (Quiñones, 2013).

El objetivo de la investigación fue el de conocer la percepción de los agentes económicos y sociales involucrados, y de los expertos, respecto a:

- La necesidad de dicha propuesta

- La viabilidad de dicha propuesta

- Las condiciones requeridas para aplicar dicha propuesta.

\section{METODOLOGÍA}

Se aplicaron encuestas a tres grupos de informantes: el sector social (gremios sindicales, asociaciones de cesantes y jubilados, ONGs relacionadas); expertos (de las principales universidades de Lima) y gestores de fondos de pensiones, los cuales respondieron en forma limitada.

Los resultados obtenidos, obviamente, no son definitivos, pues su carácter académico no pasa en ese nivel a la aplicación, pero indican claramente el sentido de los cambios a realizar.

\section{RESULTADOS}

Las encuestas aplicadas a los tres grupos de informantes produjeron los resultados que se sintetizarán en la parte final del presente artículo. Pero es útil reseñar algunos resultados específicos, que son de gran pertinencia e impacto en el estudio.

Una pregunta de trascendencia fue sobre si se consideraría positivo que la ONP pueda invertir sus fondos previsionales en el Mercado de Valores (al igual que lo hacen las AFPs). Para tal caso, el 60\% respondió afirmativamente, el $20 \%$ en duda y el $20 \%$ restante, en forma negativa, tal como se datalla en la Figura No 01. (Siguiente página)

Siguiendo con ese aspecto, se preguntó a los informantes, si de darse la Inversión en portafolio de fondos previsionales gestionados por la ONP, se obtendría una rentabilidad similar a la de los fondos de las AFPs. Para ello, el $40 \%$ mostró una respuesta positiva, un $40 \%$ estuvo en duda y el $20 \%$ restante, negativa, tal como se detalla en la Figura No 02. (Siguiente Página) 
Figura No 01

\section{¿CREE USTED QUE SERÍA POSITIVO QUE LA ONP PUEDA INVERTIR SUS FONDOS PREVISIONALES EN EL MERCADO DE VALORES?}

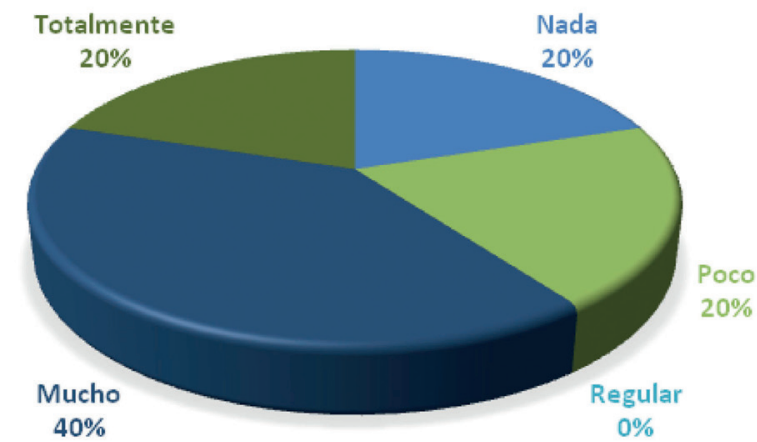

Fuente: Elaboración propia.

Figura No 02

\section{¿CONSIDERA QUE LA INVESTIGACIÓN EN PORTAFOLIO DE FONDOS PREVISIONALES GESTIONADOS POR LA ONP TENDRÍA UNA RENTABILIDAD SIMILAR AL DE LOS FONDOS DE LAS AFP?}

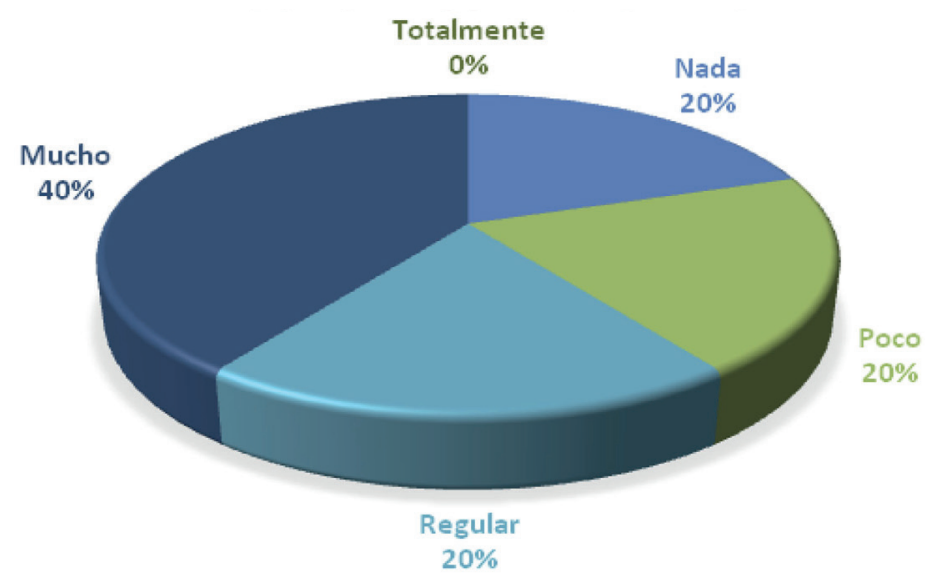

Fuente: Elaboración propia.

Se muestra así, que hay potencial para generar inversiones de la ONP en el mercado de valores, siendo consideradas como posibles y positivas.

Respecto a la posibilidad de incrementar la oferta de valores para los fondos de la ONP en el mercado de valores, se preguntó por el potencial de emisión de instrumentos de inversión públicas (guberna- mentales), teniendo como respuesta, que el $60 \%$ considera que existe un fuerte potencial para dicho fin. el $20 \%$ presentó una respuesta dubitativa, y el $20 \%$, una respuesta pesimista, tal como es visto en la Figura $\mathrm{N}^{\circ}$ 03. (Siguiente página)Concluyéndose, que sí se encuentra potencial para emitir instrumentos de inversión pública en el mercado de valores. 
Figura No 03

\section{¿CONSIDERA QUE EXISTEN POTENCIAL DE EMISIÓN DE INSTRUMENTOS DE INVERSIÓN PÚBLICAS (GUBERNAMENTALES)?}

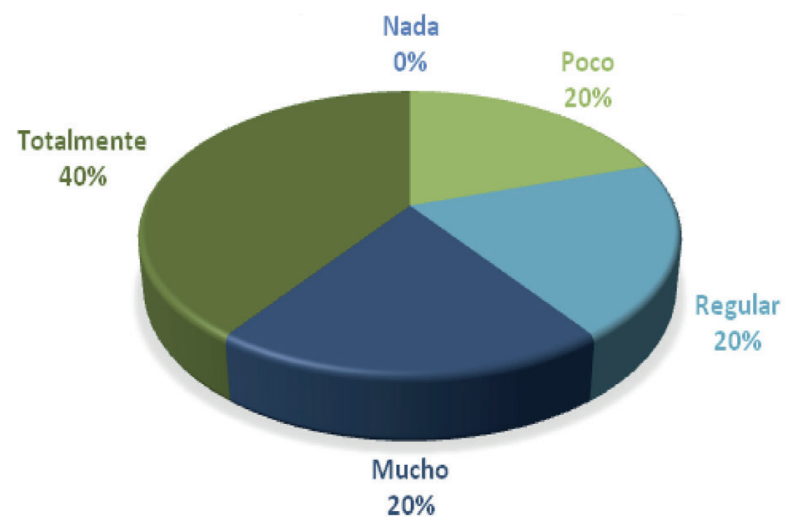

Fuente: Elaboración propia.

\section{CONCLUSIONES}

1. Según el análisis de la información, la experiencia del Sistema Privado de Pensiones SPP es discutible en relación al objetivo central, el bienestar de los trabajadores aportantes - en lo referido a la pensión otorgada y la cobertura. En el caso del objetivo secundario, el impacto en el mercado de capitales, es muy positiva en su contribución al fortalecimiento de los mercados de capitales, al incrementar la demanda de los activos financieros.

2. En el caso del sistema público de pensiones, sus resultados como sistema son muy cuestionables, en relación al bienestar de los trabajadores aportantes (pensión otorgada), la sostenibilidad del sistema y la cobertura; $y$ en el objetivo secundario de la investigación, se encuentran antecedentes de la existencia del Fondo Complementario de Reservas, la cual tuvo un desarrollo adecuado, pero que no se incrementó, limitando sus aportes positivos.

3. Por las razones expuestas, los cambios indicados son deseables y posibles, tanto por el sector social y académico entrevistado.

4. Tanto los gremios representativos de los involucrados, cómo los especialistas consideran que:

La profesionalización de las inversiones del Fondo Nacional de Pensiones, con su aplicación al Mercado de Valores peruano sería conveniente y po- sible, para incrementar la competitividad y eficiencia del sistema de pensiones, para lo cual se sugieren las siguientes condiciones:

a. Cambios normativos que permitan realizar inversiones por parte del Sistema Público de pensiones y la Oficina Nacional Previsional.

b. Sometimiento de las potenciales inversiones del Fondo Nacional de Pensiones a las normas de transparencia, disciplina y regulaciones adicionales a establecidas por la Superintendencia del Mercado de Valores.

c. Fortalecimiento e institucionalización del Mercado de Valores nacional, a través de la promoción del ingreso de más creadores de capital (empresas emisoras de valores); incluyendo, como en el caso delos Estados Unidos, al Estado, en la etapa inicial del mercado de valores.

d. El buen éxito de estas políticas estará condicionado al correcto mantenimiento de las políticas macroeconómicas, a la adecuada inversión pública, y a un marco económico internacional estable. e. La mayor competitividad y eficiencia lograda, redundará en un mayor bienestar para los afiliados y pensionistas de todo el sistema; tanto público como privado; incrementando la estabilidad del Mercado de Valores peruano y la estabilidad macroeconómica del país.

f. La posibilidad de facilitar transferencias y dar

40/ QVIPURAMAYOC | Vol. 24(46) 2016 
más incentivos para las mismas, entre AFPy ONP, acrecentándose en ambos sentidos, incrementaría la competitividad en el sistema en su conjunto

\section{REFERENCIAS BIBLIOGRÁFICAS}

1. SANCHEZ, R(et. al), 2014. Un modelo para el sistema de pensiones en el Perú: Diagnóstico y recomendaciones. 2014Banco Central de Reserva del Perú. Revista Estudios Económicos 27, 81 - 98 (Junio 2014). Disponible en www.bcrp.gob.pe/ publicaciones/revista-estudios-economicos/ estudios-economicos-no-27.html

2. CRUZ,M; MENDOZA, J;SEMINARIO, B(2014) El Sistema Previsional del Perú: Diagnóstico 1996 - 2013, Proyecciones 2014 - 2050 y Reforma. Documento de Discusión DD/14/11. Lima. Universidad Del Pacifico.

3. Defensoría del Pueblo. (2008). Informe Defensorial $\mathrm{N}^{\circ}$ 135: Por un acceso justo a la pensión: Aportes para una mejor gestión de la ONP. Lima: Defensoría del Pueblo.
4. MENDIOLA ,A; AGUIRRE,C. (2013). Análisis del sistema privado de pensiones: propuesta de reforma y generación de valor. Lima: ESAN.

5. MENDOZA, J. Y SEMINARIO, B. "El sistema previsional del Perú: diagnostico 1996-2013, proyecciones 2014-2050". Centro de Investigación - Universidad del Pacifico. 2014.

6. QUIÑONES, J(2013). Presentación sobre el Fondo Consolidado de Reservas previsionales. Recuperado en: https://www.flar.net/documentos/6348_Jose Quiñones_FCR_FLAR.ppt

7. SBS. Compendio Estadístico del SPP.Disponible en: http://www.sbs.gob.pe/app/stats/EstadisticaBoletinEstadistico.asp? $\mathrm{p}=37$ \#

8. Valdivieso Luis: 2013. "Las reformas pensionales en América Latina: el caso de Perú". En: "El Ahorro Individual Mejores Pensiones y Más Desarrollo Económico”. Federación Internacional de Administradoras de Fondos de Pensiones (FIAP). Santiago de Chile. Primera edición. 